\title{
2+1 (2+2) REMPI-TOF STUDY OF THE LOWEST RYDBERG STATES (6s, 6s') OF METHYL AND PROPYL IODIDES
}

\author{
S. COURIS and P. AGAPAKI \\ Foundation for Research and Technology-Hellas, Institute of Electronic Structure \\ and Laser, P. O. Box 1527, 71110 Heraklion, Crete, Greece
}

\author{
PAUL BRINT \\ Department of Chemistry, University College, Cork, Ireland
}

(Received 20 May, 1992)

\begin{abstract}
Resonance enhanced multiphoton ionisation (REMPI) combined with time of flight mass analysis under supersonic beam conditions has been used to investigate the nature of the UV absorption spectra broadening of the 6s Rydberg states in alkyl iodides.

The increase of the size of the alkyl substituent was associated with this broadening although it was not known whether this was due to an increase in vibronic activity or to Rydberg state substituent interaction.

The Jet cooled REMPI spectra of methyl and propyl iodide obtained have confirmed that the source of broadening of the 6s Rydberg states is solely due to vibronic activity of the alkyl group attached to the spectroscopic chromophore.
\end{abstract}

KEY WORDS: Resonance enhanced multiphoton ionisation, alkyl iodides, Rydberg states, molecular beam.

\section{INTRODUCTION}

The absorption spectrum of methyl iodide in the low energy region of the vacuum ultraviolet $(170-200 \mathrm{~nm})$ has been the subject of numerous one-photon absorption and multiphoton ionization studies. ${ }^{1,2,3,4,5}$ This interest derives from the very sharp, clear groups of vibronic lines associated with the electronic states arising from the lowest energy Rydberg transition of the molecule that are found in this region. The details of these absorptions are well understood and the observed absorptions are fully assigned. Our interest in methyl iodide is as a model for the larger alkyl iodides. We are particularly interested in the nature of the interaction between Rydberg states and substituents attached to the spectroscopic chromophore, and the iodides are an excellent case for study as the precursor molecule, methyl iodide, exhibits such a clear spectrum. 
The corresponding spectra of any higher iodide shows far broader structure, and from our earlier work on ketone compounds ${ }^{6}$ we are confident that there are two sources of this broadening, either increased vibronic activity in the spectrum, complicating the absorption band, or direct physical interaction of the Rydberg orbital with the chromophore resulting in band broadening in a manner similar to the effect of a high pressure of an inert gas perturber. If the former is the source of the more complex absorption bands then cooling the molecule to low temperatures in a molecular jet should remove some or all of the structure, if the latter, then cooling should have little effect.

The one photon absorption spectrum of methyl iodide has been discussed in detail and a low resolution overview of the higher iodides has been presented. ${ }^{2}$ The method of choice for this study, however, is resonance enhanced multiphoton ionisation. The REMPI spectrum of methyl iodide in a static cell at room temperature has been reported, ${ }^{4}$ but provided little information additional to the absorption data. This work also presented REMPI data on some higher iodides, but these were also no improvement on the available absorption data. Very recently the methyl iodide spectrum has been measured $^{5}$ for a low temperature sample using molecular jet cooling and massselecting, by time-of-flight, the ion whose intensity is monitored against the ionisation wavelength. This is exactly the experiment we have employed and our results are very similar. We repeat the data, briefly, in order to make clear the comparison with a higher iodide, propyl iodide. There have been no previous REMPI studies on any higher iodides in a molecular jet.

\section{EXPERIMENTAL}

The apparatus used in this study is essentially identical with that reported previously by the authors. ${ }^{6}$ A Lambda Physik EMG 201 MSC XeCl excimer laser pumped a Lambda Physik FL 2002 dye laser. The laser radiation was focused by means of a $15 \mathrm{~cm}$ focal length lens, to intersect a supersonic beam from a Laser Technics pulsed nozzle, $0.3 \mathrm{~mm}$ diameter. Ions produced from the multiphoton ionisation process were repelled by a $600 \mathrm{~V} \mathrm{~cm}^{-1}$ field into a $60 \mathrm{~cm}$ time-of-flight mass spectrometer and were detected by a pair of channel plates. The gate of an SRS 250 boxcar averager was positioned on the mass of the ion of interest under study and the output of the boxcar was fed in a PC-based A/D converter. The PC was not only ensuring the timing between the laser, the opening of the pulsed valve and the collection of the data for further analysis, but it was also controlling the scanning of the dye laser. The spectral region between 350 up to $405 \mathrm{~nm}$ was covered using DMQ, QUI and PBBO dyes. The wavelengths were calibrated from known transitions in $\mathrm{Kr}, \mathrm{Xe}$ and $I_{2}$ which span the region of interest. The error on the absolute energies of the peak maxima is of the order of $3 \mathrm{~cm}^{-1}$.

The TOF mass spectrometer has a mass resolution of about 250 , sufficiently large for the needs of our experiments. In all the experiments the boxcar gate selected the ions of the parent molecule $\mathrm{CH}_{3} \mathrm{I}^{+}(\mathrm{m} / \mathrm{q}=142)$. $\mathrm{The} \mathrm{CH}_{3}^{+}(\mathrm{m} / \mathrm{q}=15)$ and $\mathrm{I}^{+}(\mathrm{m} / \mathrm{q}=$ 
127) ions were also detected in the mass spectra depending on the laser wavelength and intensity.

The methyl and propyl iodide were purified by several vacuum freeze thaw cycles and were seeded with up to $5 \mathrm{~atm}$ of $\mathrm{He}$ in order to achieve sufficient vibrational cooling.

\section{RESULTS AND DISCUSSION}

The states involved in the absorption spectrum of methyl iodide are those arising from the lowest energy Rydberg configuration $(5 \mathrm{p} \pi)^{3}(6 \mathrm{~s})^{1}$ and are shown in Figure 1 in the LS coupling scheme and $\mathrm{C}_{\mathrm{oo}} \mathrm{v}$ symmetry on the left and in $\mathrm{j}-\mathrm{j}$ coupling of the core and $\mathrm{C}_{3 \mathrm{v}}$ symmetry on the right. In the linear symmetry, to which methyl iodide approximates, transitions are allowed to $\Sigma^{+}$and $\Pi$ states in one-photon absorption and to $\Sigma^{+}, \Pi, \Delta$ states in two-photon absorption. In $\mathrm{C}_{3 \mathrm{v}}$ symmetry transitions are allowed to $\mathrm{A}_{1}$ and $\mathrm{E}$ symmetry states in both processes. In one-photon absorption the spectrum is dominated by the two $\Pi$ states, with only weak vibronic lines of the $\Sigma^{+}$state evident, and there is no evidence of structure due to the $\Delta$ state even though it is technically allowed in the true symmetry of the molecule. This demonstrates the extent to which the approximate linear symmetry of the molecule controls its electronic behaviour.

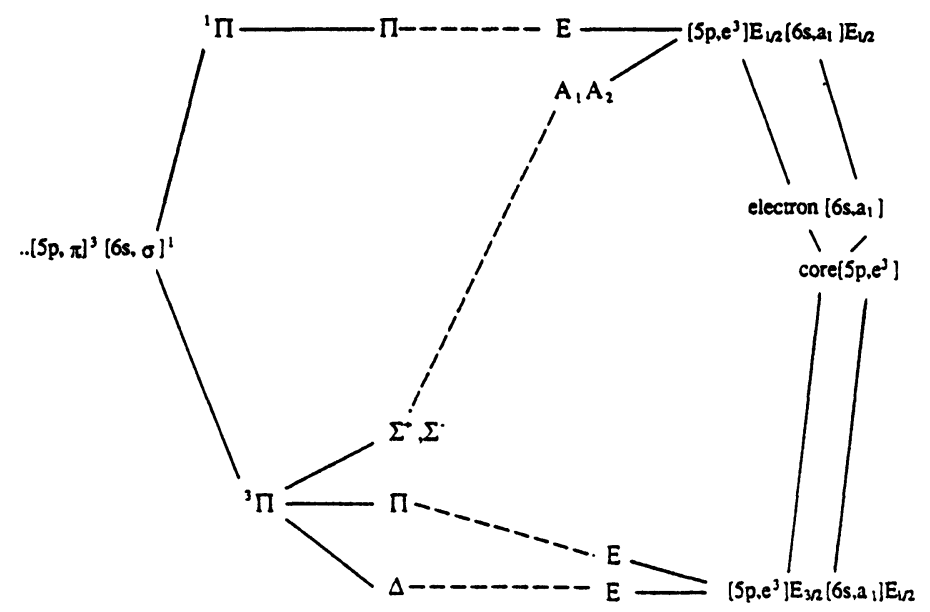

L.S COUPLING
IN LINEAR SYMMETRY

$\left.\operatorname{liN}_{\text {INere }} \mathrm{C}_{3 \mathrm{v}} \mathrm{j}^{\mathrm{j} \text { electron }}\right]$ COUPLING

Figure 1 Energy level diagram of states arising from the $(3 \mathrm{p} \pi)^{3}(6 \mathrm{~s})^{1}$ Rydberg configuration as a function of spin-orbit coupling from the LS limit to the $\Omega_{\mathrm{c}} \omega$ limit. 
The REMPI spectrum, both in a static cell and molecular jet apparatus show one major difference. The transition to the $\Sigma^{+}$state is now the strongest line in the spectrum. Only the origin line of this transition carries significant oscillator strength, unlike the two $\Pi$ states which exhibit extensive vibronic structure. The spectrum is shown in Figure 2 and is identical to that recently reported using the same measurement method. We agree with all the vibrational assignments of that paper and therefore do not discuss them here, simply listing our observed transition energies in Table 1. We did not, however, observe any additional structure in the spectrum due to the polymeric species discussed in the previous report. ${ }^{5}$

Table: Vibrational analysis of $\mathrm{CH}_{3}$ I MPI spectrum of $6 \mathrm{~s} \leftarrow 5 \mathrm{p} \pi$ molecular Rydberg transitions. ( $\lambda$ : is the laser wavelength; $2 / \lambda$ is the two photon energy; $X$ : $Y_{i}^{j}, X$ refers to the excited electronic state, $y$ to the vibrational mode and $i$ and $j$ to the number of quanta in the ground and excited states respectively)

\begin{tabular}{llll}
\hline$\lambda(\mathrm{nm})$ & $2 / \lambda\left(\mathrm{cm}^{-1}\right)$ & $\Delta \nu\left(\mathrm{cm}^{-1}\right)$ & Assignment \\
\hline 402.21 & 49725 & 0 & $\Pi: 0-0$ \\
398.22 & & 498 & $\Pi: 3_{0}^{1}$ \\
395.59 & & 832 & $\Pi: 6_{0}^{1}$ \\
393.57 & 1091 & $\Pi: 2_{0}^{1}$ \\
392.29 & 1257 & $\Pi: 5_{0}^{1}$ \\
389.81 & 1582 & $\Pi: 6_{0}^{2}$ \\
387.87 & 1839 & $\Pi: 2_{0}^{1} 6_{0}^{2}$ \\
385.48 & & $\Pi: 2_{0}^{2}$ \\
382.1. & 2158 & $\Pi: 1_{0}^{1}$ \\
380.51 & & 2836 & $\Pi: 4_{0}^{1}$ \\
370.28 & & 4289 & $\Pi: 2_{0}^{1} 4_{0}^{1}$ \\
370.00 & 0 & $\Sigma^{+}: 0-0$ \\
365.92 & 0 & $\Pi: 0-0$ \\
364.42 & & $\Sigma^{+}: 6_{0}^{1}$ \\
362.64 & & $\Sigma^{+} 2_{0}^{1}$ \\
360.41 & 54053 & 1098 & $\Pi: 6_{0}^{1}$ \\
358.84 & 54656 & 1079 & $\Pi: 2_{0}^{1}$ \\
357.58 & & 1276 & $\Pi: 5_{0}^{1}$ \\
355.63 & & 1581 & $\Pi: \sigma_{0}^{2}$ \\
352.07 & 2150 & $\Pi: 1_{0}^{1}$ \\
348.82 & & 2680 & \\
\hline
\end{tabular}

Figure 3 shows the equivalent REMPI spectrum of propyl iodide. It is immediately obvious that the two $\Pi$ transitions now exhibit far more complex and broader FrankCondon patterns. This is also true for the one-photon absorption and the static cell REMPI spectra of this molecule. Molecular jet cooling has improved the resolution of this spectrum to some extent, a fact we established by measuring the spectrum under a variety of conditions, representing a variety of different degrees of cooling, and observed narrowing and simplification of the structure, but not to the extent that would really facilitate analysis and assignment. One reason for this is that in the 


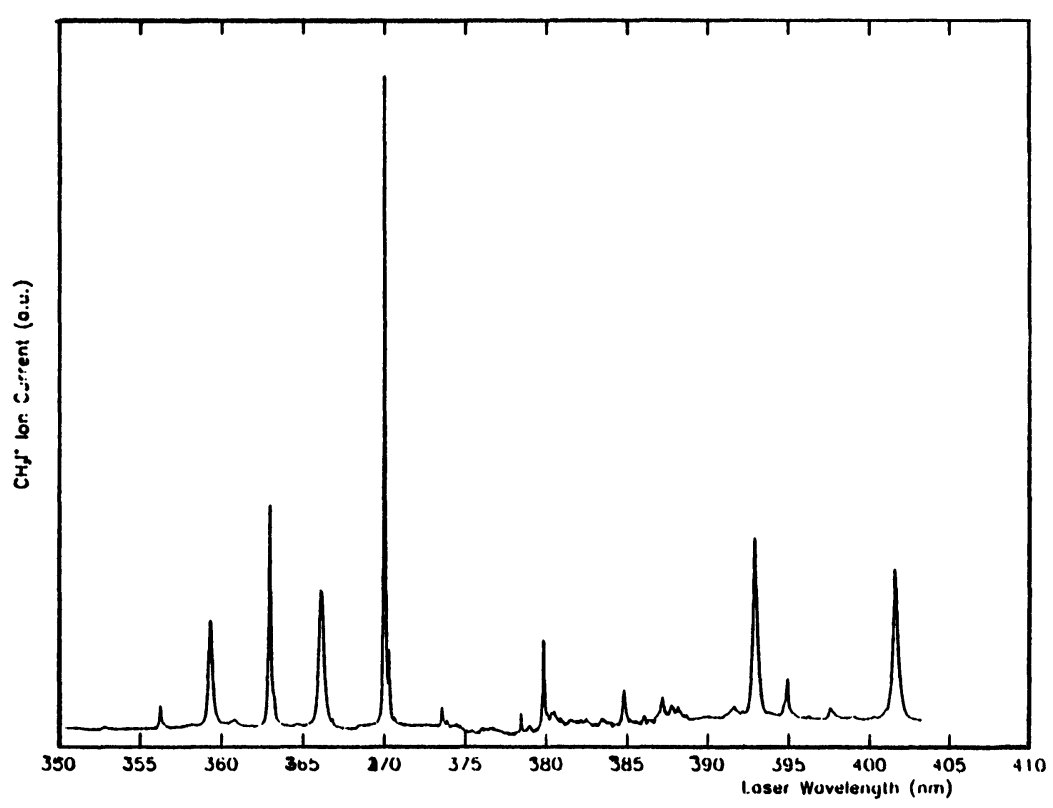

Figure 2 REMPI spectrum of methyl iodide.

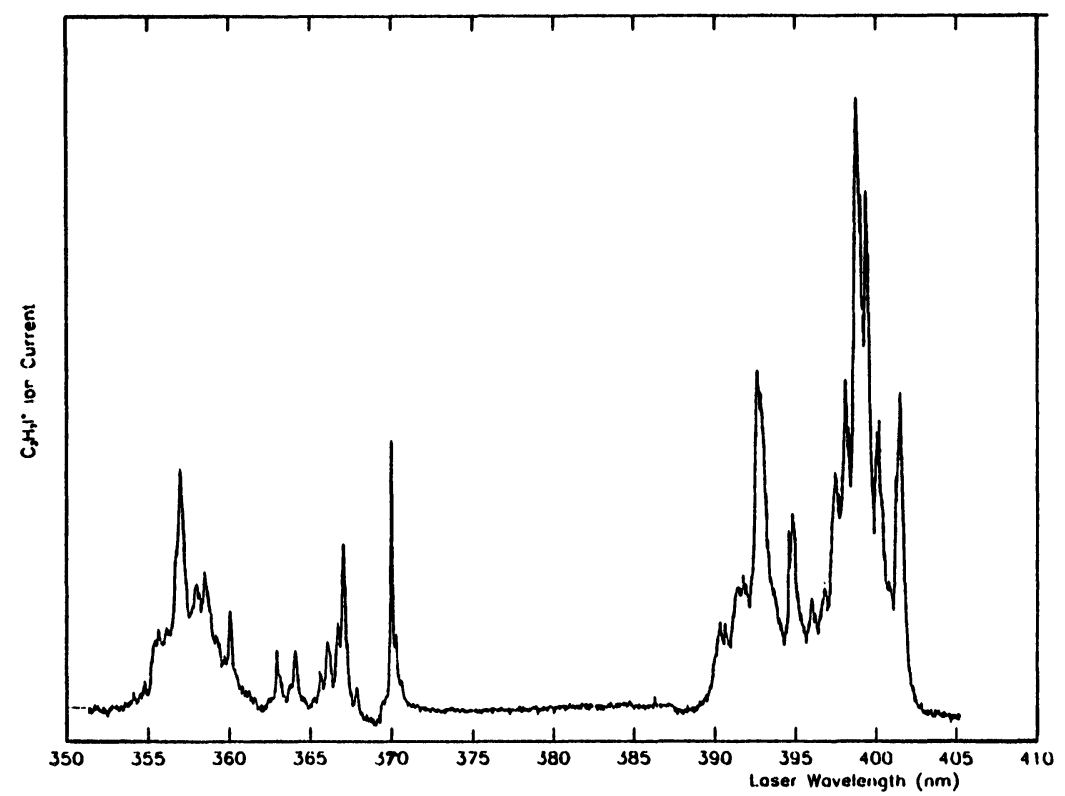

Figure 3 REMPI spectrum of propyl iodide. 
low $\mathrm{C}_{\mathrm{s}}$ symmetry of propyl iodide $\Pi$ states split into two non-degenerate electronic states of $\mathrm{A}^{\prime}$ and $\mathrm{A}^{\prime \prime}$ symmetry and therefore each of these broad band systems are really two overlapping broad transitions. A further complication is that all vibrational modes are non-degenerate and allowed, and therefore a large number of vibronic transitions are possible. This is one of the two sources of broadening of Rydberg transitions mentioned above, increased vibronic activity, and certainly appears to be the dominant process for these $\Pi$ states transitions. We make no attempt to analyse these bands. There are clearly complicated by some very low energy motions of the propyl group, probably $\mathrm{CH}_{3}$ torsional motions, that are occupied even at the low temperature of the jet.

The major feature of this spectrum is that the $\Sigma^{+}$transition is still a single sharp line, with any associated weak vibronic structure masked by the adjacent $\Pi$ transition, exactly as it was in the parent compound. The maximum of this peak is at exactly the same wavelength as the corresponding peak in the methyl iodide spectrum. This initially lead us to suspect that it may possibly be due to an impurity in both compounds. However we are now certain that it is simply a coincidence. It is not due to a methyl iodide impurity in the propyl iodide as none of the other peaks of that compound are observed. It is not due to a third compound, a common impurity to the two iodides, as we monitored different molecule, i.e. with distinctive alkyl component. On large scale expansion of the band in the two spectra there are distinctive differences on the rotational structure of the band envelope that show that there are not the exact same transition. The coincidence in energy is a genuine coincidence.

The source of broadening of Rydberg transitions, which was our original interest in this spectrum, is almost certainly simply vibronic activity. This is not surprising as the low energy Rydberg orbital is also the smallest and the one least likely to interact with the alkyl substituent. Further work, both absorption and REMPI, is needed on higher energy transitions to see how far this extends and where the other mechanism of Rydberg-substituent interaction becomes the more important. There is also the possibility that the broadening is due to mixing with a similar energy molecular state, but there is no direct evidence for this. More interesting now is the distinctive character of the $\Sigma^{+}$transition. As the $\Sigma^{+}$state arises from the same electron configuration as the $\Pi$ states, and is also a bound state, one would not expect it to have a very different potential energy surface. It is different, as the methyl iodide spectrum shows, the transition has a sharper Frank-Condon pattern suggesting a potential energy surface very similar to the ground state, but not very different as the $\Pi$ transitions are fairly sharp. Why this distinction should persist, in fact become exaggerated, in the propyl iodide molecule where, quite clearly, the $\Pi$ states have potential energy surface quite different to the ground state but the $\Sigma^{+}$state is still very similar is not clear. Considerably more work on these and related molecules is indicated.

P.B. gratefully acknowledges support from the "UV Laser Facility" operated in FORTH-IESL (Crete) within the "Large Installations Plan" of the European Community. 


\section{References}

1. R. S. Mulliken. Phys. Rev., 61, 277 (1942).

R. S. Mulliken and E. Teller. Phys. Rev., 61, 283 (1942).

2. R. A. Boschi and D. R. Salahub. Molec. Phys., 24, (2), 289 (1972).

3. S. Felps, P. Hochmann, P. Brint and S. P. Mc. Glynn. J. Mol. Spectrosc., 59, 355 (1976).

4. D. H. Parker, R. Pandolfi, P. R. Stannard and M. A. EI-Sayed. Chem. Phys., 45, 27 (1980)

5. V. Vaida, D. J. Donaldson, S. P. Sapers and R. Naaman. J. Chem. Soc. Faraday Trans., 86 (11), 2043 (1990).

6. P. Brint, L. O'Toole, S. Couris, D. Jardine. J. Chem. Soc. Faraday Trans., 87 (18), 2891 (1991).

7. J. D. Scott, W. S. Felps, G. L. Findley and S. P. Mc. Glynn. J. Chem. Phys., 68 (10), 4678 (1978). 